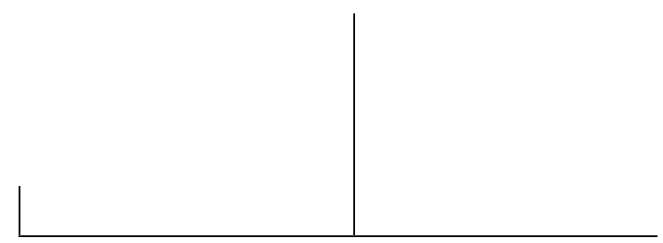

Rev. Latinoam. Psicopat. Fund., São Paulo, 16(1), 56-70 mar. 2013

\title{
Psicopatología psicoanalítica: un saber en la encrucijada*
}

Miguel Angel Sierra Rubio

Con el objetivo de esclarecer la condición epistémica de la psicopatología psicoanalítica en el marco del saber freudiano, se analizan cuestionamientos previos sobre el campo psicopatológico y sus posibles modalidades relacionales con el psicoanálisis, la psicología clínica y la psiquiatría. Los resultados destacan la originalidad de la estrategia de Freud que, bajo el nombre del psicoanálisis, cobija un discurso racional sobre la experiencia del malestar subjetivo.

Palabras clave: Saber freudiano, psicopatología, psicoanálisis, epistemología

* Una versión preliminar de este trabajo fue presentada en el II Congreso Internacional de Psicoanálisis en la Universidad Autónoma de Querétaro (México), en septiembre de 2011. 


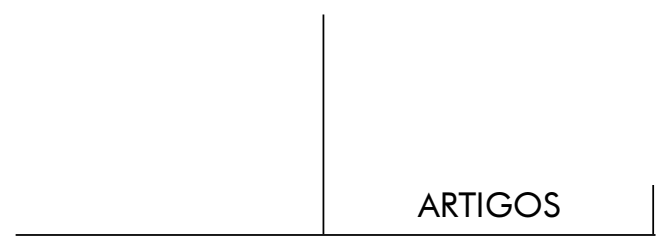

\section{Introducción}

De acuerdo con Samacher (2005), se debe a Moritz la acuñación, en 1783, del término psicopatología para designar un campo que había sido la heredad de ministros religiosos, hechiceros e incluso filósofos. El término fue perimido, hasta que sucesivas generaciones de médicos del siglo XIX hicieron suya la parcela, bautizándola como psiquiatría clínica y estableciendo posteriormente como sinónima la expresión psicopatología general (Emminghaus, 1878). En la actualidad, la psicopatología constituye la base de la psicología clínica y de la psiquiatría en tanto aplicaciones a la salud mental, aunque es considerada como entidad distinta de ellas.

Fecundo en labradores, el campo psicopatológico es también sitio de encrucijada para el psicoanálisis, pues lo coloca en una situación difícil. Por una parte, existen afirmaciones tajantes en el sentido de que la psicopatología constituye "el terreno originario y fundamental del pensamiento psicoanalítico" (Cahn, 2003, p. 453); por otra, hay analistas que, como Casanova (1998), sostienen sin vacilar su mutua exclusión. Asistimos aquí a una curiosa repetición de la historia: el primer órgano científico especializado en la difusión de la Ciencia del Inconsciente, el Jahrbuch für psychoanalytische und psychopathologische Forschungen, ${ }^{1}$ que inicialmente tenía por editores a la dupla Freud-Bleuler y como redactor a Jung, omitió en su rótulo principal la referencia a la investigación psicopatológica a partir de 1914, luego de la ruptura de los suizos con el Psicoanalista de Viena. La revista sobrevivió sólo para un último volumen, antes del inicio de la Primera Guerra Mundial.

${ }^{1}$ Anuario de Investigaciones Psicoanalíticas y Psicopatológicas. 
Así, el gesto freudiano que por un lado atribuye al psicoanálisis un lugar cercano al territorio de la psicopatología, y por el otro hace exclusión nominal de ella, vuelve perturbador y enigmático el sintagma psicopatología psicoanalítica. Por eso es legítimo preguntarnos: ¿Qué puede significar en estos tiempos tal expresión sintagmática? Con el objetivo de esclarecer la condición epistémica de la psicopatología psicoanalítica en el marco del saber freudiano, haremos un rodeo por cuestionamientos previos sobre el campo psicopatológico y sus posibles modalidades relacionales con el psicoanálisis, la psicología clínica y la psiquiatría.

\section{El estatus epistémico del campo psicopatológico}

La historia intelectual de Occidente en los siglos XIX y XX se caracteriza por la atomización del conocimiento, mediante la creación de disciplinas autónomas que recortan lo real ${ }^{2}$ para construir sus objetos de estudio; y que, al alcanzar cierto progreso científico, ven subdividido su tronco disciplinario central en ramas o especialidades. Así, tanto la psicología clínica como la psiquiatría se consideran comúnmente ramas disciplinarias: la primera, de la psicología; la segunda, de la medicina.

En lo que concierne a la psicopatología, los inicios de su reconocimiento como entidad autónoma se remontan cuando menos a Jaspers (1913), quien en su Psicopatología general establecía el deslinde entre: la psiquiatría como profesión práctica de cuidado, vigilancia, tratamiento y peritaje sobre casos individuales; la psicología como estudio de la vida psíquica normal; y la psicopatología como ciencia del acontecimiento psíquico patológico. La promoción de esta psicopatología general como disciplina independiente - bien sea que se suscriba la perspectiva fenomenológica jasperiana o no (Esteban, 2005) - continúa hasta la fecha, aunque no sin querella.

Monedero (1996), por ejemplo, denuncia que la Psicopatología "se ha visto alienada en la Psiquiatría desde sus comienzos" (p. 10) al figurar bajo el nombre

${ }^{2}$ De acuerdo con Brugger (1978), la noción de real denota "lo que es, lo que existe en oposición tanto a lo meramente aparente como a lo puramente posible" (p. 439). En este trabajo se le encontrará en forma sustantivada neutra - lo real -, y bajo el supuesto hipotético de su independencia con respecto a la racionalidad científica que lo dispone o recorta en múltiples porciones - objetos de conocimiento de las disciplinas. Como tal, esta noción es congruente, entre otros, con los desarrollos de Zemelman (1998) y Wallerstein (1996) sobre la configuración disciplinaria de la ciencia. 


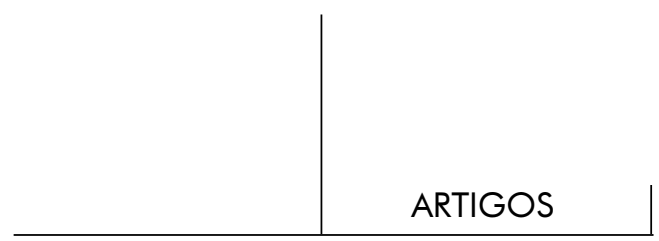

de psiquiatría general en los manuales de esta rama de la medicina. Un destino similar habría enfrentado entre los psicólogos estadounidenses, que serían responsables de la alienación de la psicopatología en el capítulo denominado psicología anormal. De esta suerte, la psicopatología habría sido reducida injustamente a mero contenido de una rama disciplinar, cuando debería brillar con el resplandor de una ciencia autónoma.

Tizón (1978), por otra parte, hace una lectura inversa de la situación. El drama de la psicopatología consistiría en que ella ha tenido, sobre todo a partir de Jaspers, un rumbo independiente de la psicología; aunque por derecho se trata de una de sus ramas, ${ }^{3}$ y su religación epistémica debería incluso inspirarnos a reescribirla de un modo que enaltezca su unión: psico(pato)logía. Reubicando la psicopatología como especialidad de la psicología, el autor pretende otorgarle credenciales de cientificidad.

Posiciones tan disímiles como las mencionadas son representativas de cierta in-identidad epistémica de la psicopatología, que se debate entre tres condiciones posibles:

a) Una psicopatología psiquiátrica, que no sería sino un contenido intradisciplinar de aquella especialidad médica.

b) Una psicopatología psicológica. O sea, una rama de la psicología, junto a la psicología clínica y otras especialidades disciplinares.

c) Una psicopatología, a secas - o, si se prefieren los términos de Emminghaus y Jaspers, una psicopatología general. Es decir, una disciplina autónoma frente a la psicología y la medicina, aunque en vínculo con ellas.

Cada estatus atribuible a la psicopatología implica una manera diferente de pensar sus relaciones con las otras entidades en juego. Si partimos del planteamiento (a), el que menor envergadura epistémica adjudica al campo psicopatológico, podemos suponer un aumento de su importancia y de las propiedades que se le atribuyen, a medida que se hace el recorrido a través de (b) hacia (c). Propongo justamente esa secuencia como interpretación posible de la constitución de ciertas entidades disciplinarias. Creo que en el paso de (a) hacia (b) podríamos localizar el origen de la psicología clínica, debido a cierta insatisfacción con los planteamientos psicopatológicos exclusivamente médicos (Belloch, 2008). El pasaje de (b) hasta (c), por otra parte, podría constituir un

${ }^{3}$ Piénsese, por ejemplo, en la psicología patológica promovida por la escuela francesa de Ribot y Janet, cuyos continuadores se extienden hasta nuestros días (Reuchlin, 1991). Agradezco a J. L. Rivera por ponerme en la pista de esta referencia. 
intento de salvar cierta trifulca teórica entre las otras entidades disciplinarias por apropiarse la psicopatología. Tal acto de independencia epistémica conllevaría igualmente la intención de que el campo psicopatológico adquiriera cierta madurez.

Pero, fundamentalmente, esta secuencia nos servirá para abordar el sintagma psicopatología psicoanalítica. Suponiendo para el psicoanálisis la dignidad epistémica de disciplina autónoma, ¿cuál es el estatus del campo psicopatológico con relación a esta ciencia del Inconsciente? Es decir: ¿Habría, como en (a), una especialidad psicoanalítica que contara entre sus contenidos a la psicopatología psicoanalítica? O ¿se trataría, como en (b), de una rama disciplinar del psicoanálisis? O ¿sería mejor pensar que, como en (c), es el resultado de un cruce entre dos disciplinas autónomas (psicoanálisis y psicopatología general)? Examinemos primeramente las cuestiones convocadas por esta última hipótesis: que el psicoanálisis y la psicopatología sean dos disciplinas autónomas que hacen encrucijada.

\section{Posibilidades en la encrucijada}

El diálogo entre ciencias autónomas encuentra en la racionalidad epistémica contemporánea al menos tres posibilidades de realización: la pluridisciplinariedad, la interdisciplinariedad y la transdisciplinariedad. Para caracterizarlas, estableceré una analogía entre cada modalidad relacional con uno de los tres procedimientos sintácticos mediante los cuales se forman oraciones compuestas en castellano (Munguía, Munguía \& Rocha, 2000): la yuxtaposición, la coordinación y la subordinación.

Comencemos por la pluridisciplinariedad. También llamada multidisciplinariedad, consiste en una mera asociación de saberes al servicio de una finalidad común: el esclarecimiento del objeto de alguno de ellos. La estructura de esta vinculación es análoga al procedimiento gramatical de yuxtaposición, en el cual dos o más oraciones simples se enlazan por el mero empalme de sus elementos con ayuda de un signo de puntuación. Nicolescu (1998) proporciona un ejemplo de estudio pluridisciplinario que tiene una estructura similar a la oración yuxtapuesta: "un cuadro del Giotto puede ser estudiado por la observación de la historia del arte cruzada con la de la física, la química, la historia de las religiones, la historia de Europa y la geometría" (p. 34). Se trata, pues, de empalmar los saberes, poner uno al lado de otro los puntos de vista disciplinarios sobre el objeto, de modo que pueda entenderse desde varias de sus aristas. De esta manera, entidades como el psicoanálisis, la psicología clínica, la psiquiatría y la psicopatología general pueden ser convocadas para el estudio de un mismo ob- 


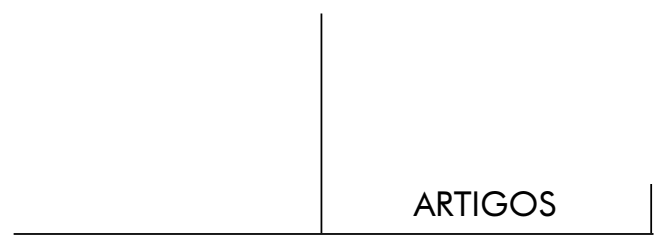

jeto, sin que por ello sean modificadas en sus fronteras epistémicas, en sus metodologías y en sus perspectivas sobre la porción de lo real que les compete. Así, la lógica de la pluridisciplinariedad imposibilita hablar de psicopatología psicoanalítica; se trataría más bien de sumar las perspectivas de dos disciplinas - la psicopatología general y el psicoanálisis - sobre un mismo objeto de estudio.

La interdisciplinariedad es una segunda opción en la encrucijada. Actualmente hay distintas versiones de ella, con mayor o menor consciencia de sus credenciales de nacimiento - los reclamos estudiantiles de 1968 - y con diversos grados de fundamentación en la ideología o en la epistemología; sin embargo, todas sus formas tienen en común el llamado a superar la división disciplinaria. Subyace aquí el postulado de que para toda disciplina hay un excedente de lo real que escapa a sus límites, por lo cual se haría necesario un espacio explicativo de articulación o rearticulación con otras. Tal discurso prioriza la sinergia del grupo interdisciplinario de investigación por sobre el esfuerzo de una sola disciplina; en una situación análoga a la coordinación sintáctica de oraciones simples, donde cada oración coordinada conserva su independencia, pero se aporta un significado adicional mediante un nexo coordinante.

En nuestro medio, resalta como interdisciplinaria la idea de la psicopatología fundamental, lanzada por Fédida en los últimos decenios del siglo XX. Esta propuesta concibe un lugar vacío en el orden del saber, "donde una pluralidad de puntos de vista en relación al pathos advienen y se transforman por obra de su propia puesta en relación y movimiento" (Singer, 2000, p. 116). Tal dispositivo no promueve una sumatoria de saberes sobre el campo psicopatológico, sino cierta tensión dialéctica de los saberes interdisciplinarizados para sostener su operación de conjunto. Ahora bien, ¿existe equivalencia lisa y llana entre tal psicopatología fundamental y la psicopatología psicoanalítica? No, desde el momento en que "la Psicopatología Fundamental sólo es psicoanalítica porque, a pesar de los psicoanalistas, hay en la casa del psicoanálisis un lugar para ella" (Berlinck, 1998, p. 58). En todo caso, lo que puede afirmarse es que la psicopatología psicoanalítica - esa cuyo estatus epistémico permanece todavía incierto en este punto de nuestro recorrido - tiene la posibilidad y el derecho de quedar concernida por el espacio interdisciplinario de la psicopatología fundamental, siempre y cuando no se coloque "como único discurso válido para tratar lo psicopatológico, perdiendo así el factor vigorizante de la dialéctica y del debate con interlocutores externos" (Costa, 1998, p. 72)

Un último procedimiento gramatical para formar oraciones compuestas es la subordinación, donde una o varias oraciones simples quedan subsumidas por otra, desempeñando en su interior funciones complementarias. El discurso transdisciplinario, estructurado a semejanza de este procedimiento, es solidario de 
cierta globalización del conocimiento, que proclama la unión orgánica de diversas entidades disciplinarias "en relación con un objeto nuevo no abarcado por ninguna de ellas" (Follari, 2001, p. 41). Es posiblemente el caso de disciplinas híbridas como la bioquímica, la geofísica, la mecatrónica, la psicopedagogía, la sociobiología... y el freudomarxismo, que Casas (2002) ha analizado como prototipo de subordinación entre disciplinas. Por su parte, una psicopatología psicoanalítica en este sentido transdisciplinario, sería una mésalliance $e^{4}$ entre el psicoanálisis y la psicopatología general, cuya fusión promovería un saber en el que ninguna de las partes podría reconocerse.

Deslindemos, de estas tres posibilidades de encrucijada, el sentido que actualmente puede tener la expresión psicopatología psicoanalítica. Porque justamente ella no es un ámbito pluri, ni inter, ni transdisciplinario, definirla a partir de este tipo de encuentro entre psicoanálisis y psicopatología general no da cuenta de lo que, freudianamente hablando, significa psicopatología psicoanalítica, ni permite captar la epistemología inmanente a su vínculo con la psicología clínica y la psiquiatría. Entonces, es momento de dejar atrás el planteamiento (c), y lanzar nuestra mirada sobre (b) y (a).

¿Es la psicopatología psicoanalítica una rama disciplinar o especialidad del psicoanálisis? Desconozco si algún autor ha avanzado algo en tal sentido. Lo cierto es que el término psicopatología psicoanalítica brilla por ausencia en los diccionarios más afamados de la disciplina (De Mijolla, 2005; Laplanche \& Pontalis, 1993; Roudinesco \& Plon, 2008), de los cuales se esperaría definición si se tratase de una rama de este saber. Tenemos, pues, razones para descartar por el momento el planteamiento (b) como hipótesis explicativa. Aún más: tal vez el psicoanálisis no haya llegado a esta etapa de madurez en la que al tronco disciplinario central le brotan ramas. Bajo tal supuesto, habría que matizar el último planteamiento, (a), y preguntarnos si la psicopatología psicoanalítica es un simple contenido intradisciplinar - prescindiendo de si está colocado en alguna posible rama o más bien en el tronco principal del árbol. Así parece considerarla Tizón (1978) cuando la concibe como una "región de la teoría psicoanalítica" (p. 98). Por su parte, Laplanche y Pontalis (1993) admiten, al definir el psicoanálisis, que se puede distinguir en esta disciplina un nivel referente al "conjunto de teorías psicológicas y psicopatológicas en las que se sistematizan los datos aportados por el método psicoanalítico" (p. 316). Tal delimitación, piensan los autores mencionados, "reproduce, en forma más detallada, la que Freud dio" (p. 317). En realidad, la cuestión es más compleja.

${ }^{4}$ Término caro a Freud, que designa un matrimonio en condiciones desiguales o un falso enlace. 


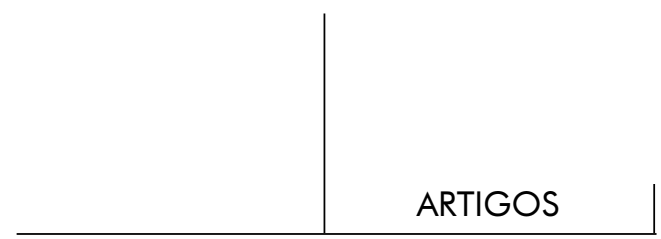

En la definición mejor perfilada que Freud (1923/2006) nos legó, no existe inicialmente una referencia explícita al campo psicopatológico. Aquello que Laplanche y Pontalis creen reproducir con más detalle no es sino una interpretación del texto freudiano que delimita la $3^{\text {a }}$ acepción del psicoanálisis como el nombre "de una serie de intelecciones psicológicas (...) que poco a poco se han ido coligando en una nueva disciplina científica" (p. 231). ¿Por qué, entonces, los autores adscriben al psicoanálisis cierto contenido psicopatológico que formaría parte esencial e indiscutible de su concepto, en calidad de psicopatología psicoanalítica?

\section{Saber freudiano y psicopatología psicoanalítica}

Hay tanto una estrategia freudiana que otorga al saber psicoanalítico su congruencia interna con el campo psicopatológico, como una historia de su novedad epistémica en este terreno. Freud (1936/2006) partió del estudio de las defensas, en particular la represión, a fin de "espigar de la psicopatología la ganancia para la psicología normal" (Freud, 1994, p. 131). El año 1895 marca, de hecho, el punto de consagración de Freud a la psicopatología. No hablamos hiperbólicamente, pues consagración es el ámbito semántico preciso que el Vienés estipuló para calificar, en retrospectiva, la contribución a los "Estudios sobre la histeria" por parte de su antiguo colaborador recién fallecido, Josef Breuer: "se consagró a la psicopatología [aunque] sólo durante un breve episodio de su larga vida" (Freud, 1925a/2006, p. 300). En el caso de Freud, su dedicación propia se manifiesta en la acuñación de una categoría conceptual comprehensiva de los actos psíquicos más heterogéneos: las formaciones psicopatológicas.

La idea fija histérica, la representación obsesiva y la idea delirante - entidades claramente patológicas en términos de la neurología y la psiquiatría de la época - pertenecen a este conjunto conceptual; pero de igual manera el chiste, el olvido, el acto fallido, el lapsus linguae y el sueño - fenómenos cotidianos y normales. Lo sorprendente es que el último de ellos se convierte, además, en el paradigma explicativo de lo anormal: "el sueño pasa a ser el arquetipo normal de todas las formaciones psicopatológicas" (Freud, 1913/2005, pp. 175-176). De la psicopatología de la vida cotidiana (Freud, 1901/2004) a la "psicopatología de las neurosis" (Freud, 1905/2005, p. 8), el común denominador teórico es el conflicto "entre una moción conciente y una reprimida" (Freud, 1942/2005, p. 280), fondo explicativo de sus fenómenos por igual. La psiquiatría de ese momento no llegaría a discernir adecuadamente esa novedad, salvo el grupo suizo de Abraham, Jung y Bleuler. En ellos piensa el Vienés cuando anuncia optimista: 
"a los psicopatólogos se les ha vuelto inevitable trabajar con procesos anímicos, representaciones, etc., inconcientes" (Freud, 1910/2006, p. 209).

Tenemos, pues, un psicoanálisis caracterizado como indispensable para la psicopatología. Encontramos la llave para objetivar su fundamento en las cartas de Freud con Jung. En efecto, el 6 de junio de 1907, el Vienés escribió: “¿Se arriesga usted a tomar en serio la lucha por el reconocimiento de nuestras innovaciones? Entonces, lo inmediato sería fundar una revista, por ejemplo, de 'Psicopatología y Psicoanálisis', o más descaradamente, tan sólo de 'Psicoanálisis"” (Freud \& Jung, 1978, p. 96). A partir de aquí, habrá dos actitudes diversas hacia el proyecto editorial que llegaría a constituirse con el nombre que ya conocemos, Anuario de Investigaciones Psicoanalíticas y Psicopatológicas. Mientras en Jung hay cierta tendencia a separar la psicopatología (psiquiátrica) y el psicoanálisis, sugiriendo, por ejemplo, incluir dos prólogos disciplinarios el del psicoanalista Freud y el del psicopatólogo Bleuler - en el primer número de la publicación; el Vienés, por el contrario, no hallaba la necesidad de esto, aduciendo que la justificación del Anuario sería visible en su índice, "distinto a una revista más de psiquiatría y enfermedades nerviosas" (Freud \& Jung, 1978, p. 229). En efecto, si acudimos a aquel número primigenio, encontramos como trabajo inaugural - portador de todo el peso de diferencia y novedad deseadas para la revista - el Análisis de la fobia de un niño de cinco años (Freud, 1909/1970). Es decir, un texto que se considera tanto uno de los cinco grandes psicoanálisis, como el paradigma psicopatológico de la fobia. Sospechamos, entonces, que lo novedosamente distinto es el pensamiento de Freud, que hace del psicoanálisis y la psicopatología una y la misma cosa.

Por si estos hechos no testimoniaran suficientemente la seriedad con que Freud luchaba por el reconocimiento de su innovación, al cabo de cinco años de publicación ocurrió el descaro. El deseo freudiano de que la revista fuese únicamente de psicoanálisis - sin ningún distingo o separación de la psicopatología -, adquirió expresión nominal en cuanto Jung hubo renunciado a su cargo editorial. Así, apareció el Jahrbuch der Psychoanalyse, ${ }^{5}$ aunque exhibiendo claramente su marca umbilical: "Nueva serie del Anuario de Investigaciones Psicoanalíticas y Psicopatológicas" (Freud, 1914/1970), subtítulo aclaratorio que autorizaba a continuar la numeración de los tomos a partir de donde había llegado su predecesor. Más tarde, Freud (1924/2006) contará el Anuario, con su primer nombre, entre "las publicaciones periódicas dedicadas exclusivamente al psicoanálisis" (p. 213).

${ }^{5}$ Anuario de Psicoanálisis. 


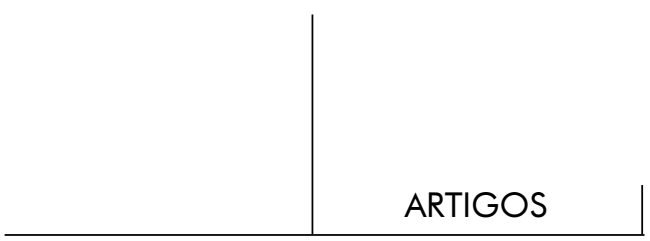

En la misma carta del 29 de noviembre de 1908 en la que el Vienés discute con Jung el asunto de los prólogos disciplinarios, se encontraba ya un presagio de la insolencia freudiana. En un momento de la epístola, Freud lanza su consigna: "ser original constituye también una meta de la vida" (Freud \& Jung, 1978, p. 227). Nosotros vemos su misión cumplida en la resuelta estrategia de cubrir bajo el nombre del Psicoanálisis a la Psicopatología, haciendo de ellos algo indistinto. Si el psicoanálisis es psicopatología, el freudismo creó, para resaltarlo, esa expresión cuyo estatus epistémico queda finalmente al descubierto: la psicopatología psicoanalítica. Laplanche y Pontalis tuvieron el acierto de reivindicar esta dimensión en su glosa del texto freudiano; lamentablemente, el formularlo bajo la noción de nivel hace pensar en una división interna de la Ciencia del Inconsciente, uno de cuyos macro-contenidos intradisciplinarios sería el conjunto psicopatológico. Más acertada resultó la aserción de Lacan (1981) según la cual "el descubrimiento freudiano asumió la forma (...) de psicopatología" (p. 389). Sin embargo, encontramos aún mayor claridad en la noción utilizada por Assoun (2006) para dar cuenta de esta circunstancia: la psicopatología psicoanalítica es una de las figuras del psicoanálisis, al lado de la clínica, la metapsicología y la técnica. No se trata, por tanto, de "una psicopatología emparejada al psicoanálisis" (Assoun, 2005, p. 128), ni de sumar "al saber psicopatológico un 'capítulo' suplementario, un agregado inconsciente a las formas de conocimiento objetivadoras del síntoma" (Assoun, 2006, p. 140). Bajo esta figura, como anunciaba Freud (1925b/2004), "el psicoanálisis deja de ser una ciencia auxiliar de la psicopatología, y es más bien el esbozo de una ciencia del alma, nueva y más fundamental, que se vuelve indispensable también para entender lo normal" (p. 44).

En su acto fundante, la psicopatología psicoanalítica se presenta como una disciplina subversiva, que se apropia la porción de lo real que otras entidades rechazan al constituirse: de la psicología, el Inconsciente; de la psiquiatría, la neurosis y la histeria. Es esta identidad transgresora lo que ha de cuidar cuando se le requiere participación en las modalidades relacionales contemporáneas.

\section{Conclusión}

El esclarecimiento de algunos de los problemas más vastos de la psicopatología, nos lleva a concluir que se trata de un campo de lo real que es susceptible de muchos modos de aprehensión racional; una especie de matriz indiferenciada que ha servido a la constitución de saberes como la psiquiatría, la psicología clínica y el psicoanálisis. Sin embargo, su aporte a la configuración de 
estas entidades ha sido diverso y no está exento de discusiones. No tomamos partido acerca de lo que debiera ser el campo psicopatológico en el conjunto actual de los saberes ni en su vinculación con la psicología clínica y la psiquiatría. Tomamos nota, sí, de lo que ha sido, y de aquello que las condiciones de la racionalidad contemporánea le marcan como posibilidad: contenido intradisciplinar, rama disciplinaria, disciplina autónoma, ámbito pluri, inter o transdisciplinario.

Con el psicoanálisis, la psicopatología adquiere una identidad epistémica singular. En nuestros tiempos, la expresión psicopatología psicoanalítica es una figura del psicoanálisis que resalta la originalidad del saber freudiano acerca del malestar subjetivo; saber esencialmente distinto a los discursos psiquiátricos y psicológicos sobre el campo psicopatológico. Para dar cuenta de lo real que le concierne, la psicopatología psicoanalítica subvierte el lenguaje proveniente de otras entidades disciplinarias - inconsciente, neurosis e histeria, por ejemplo -, y acuña un conjunto de categorías que le haga justicia - formaciones psicopatológicas, psicopatología de la vida cotidiana, entre otras.

En la racionalidad contemporánea existe un llamado a superar la estructura disciplinar, a través de diversas modalidades relacionales que hacen encrucijada. Cuando la psicopatología psicoanalítica, figura del psicoanálisis, participe en la construcción de espacios pluridisciplinarios - en yuxtaposición con la psicopatología general, por ejemplo - e interdisciplinarios - en coordinación con otros saberes en la psicopatología fundamental o en dispositivos alternos -, sólo podrá mantener su originalidad y su identidad permaneciendo alerta ante los riesgos de subordinación y fusión transdisciplinarias de los que puede ser objeto o agente. Si la psicopatología psicoanalítica quiere un porvenir, si está dispuesta a crear diálogo en la encrucijada, le es indispensable, hoy como ayer, ser fiel a la novedad epistémica que la sostiene.

\section{Referencias}

Assoun, P.-L. (2005). L'avenir sans illusions. Prolégomènes au débat sur l'avenir de la psychanalyse dans le champ de la psychopathologie. Psychologie clinique. Paris, 20, 125-129.

Assoun, P.-L. (2006). Figuras del psicoanálisis. Buenos Aires: Prometeo.

Belloch, A. (2008). Psicología y Psicología Clínica: sobre árboles y ramas. Análisis y modificación de conducta. Huelva, XXXIV(150-151), 67-93.

Berlinck, M. T. (1998, mar.). O que é Psicopatologia Fundamental. Revista Latinoamericana de Psicopatologia Fundamental. São Paulo, 1(1), 46-59.

Brugger, W. (1978). Diccionario de filosofía. Barcelona: Herder. 
Cahn, R. (2003). Neurosis, psicosis y perversiones. In De Mijolla, A. \& De Mijolla-Mellor, S. Fundamentos del psicoanálisis (pp. 453-496). Madrid: Síntesis.

Casanova, B. (1998). Estallidos de clínica. Litoral, Buenos Aires, 25/26, 107-116.

Casas, J. (2002). Relación del psicoanálisis con otros campos disciplinarios: una apreciación. Querétaro: Universidad Autónoma de Querétaro.

Costa, M. (1998, mar.). Formulando uma Psicopatologia Fundamental. Revista Latinoamericana de Psicopatologia Fundamental, São Paulo, 1(1), 60-76.

De Mijolla, A. (Ed.) (2005). International Dictionary of Psychoanalysis. Farmington Hills: Thomson /Gale.

Emminghaus, H. (1878). Allgemeine Psychopathologie zur Einführung in das Studium der Geistesstörungen. Leipzig: Vogel.

Esteban, R. (2005). Sobre algunas disciplinas fundamentales para la Psicopatología General. Norte de Salud Mental. Erandio, 6(24), 28-37.

Follari, R. (2001). Estudios culturales, transdisciplinariedad e interdisciplinariedad (¿hegemonismo en las ciencias sociales latinoamericanas?). Utopía y praxis latinoamericana. Maracaibo, 6(14) 40-47.

Freud, S. (2004). Psicopatología de la vida cotidiana. In Obras completas (v. VI, pp. 1-270). Buenos Aires: Amorrortu. (Trabalho original publicado em 1901).

Freud, S. (2005). Fragmento de análisis de un caso de histeria. In Obras completas (v. VII, pp. 1-107). Buenos Aires: Amorrortu. (Trabalho original publicado em 1905).

Freud, S. (1970). Analyse der Phobie eines 5jährigen Knaben. Jahrbuch für psychoanalytische und psychopathologische Forschungen. Nendel/ Liechtenstein, I(1), 1-109. (Trabalho original publicado em 1909).

Freud, S. (2006). La perturbación psicógena de la visión según el psicoanálisis. In Obras Completas (v. XI, pp. 205-216). Buenos Aires: Amorrortu. (Trabalho original publicado em 1910).

Freud, S. (2005). El interés por el psicoanálisis. In Obras completas (v. XIII, pp. 165-192). Buenos Aires: Amorrortu. (Trabalho original publicado em 1913).

Freud, S. (Ed.). (1970). Jahrbuch der Psychoanalyse (v. VI). Nendel/Liechtenstein: Krauss Reprint. (Trabalho original publicado em 1914).

Freud, S. (2006). Dos artículos de enciclopedia: "Psicoanálisis" y "Teoría de la libido". In Obras completas (v. XVIII, pp. 227-254). Buenos Aires: Amorrortu. (Trabalho original publicado em 1923).

Freud, S. (2006). Breve informe sobre el psicoanálisis. In Obras completas (v. XIX, pp. 199-221). Buenos Aires: Amorrortu. (Trabalho original publicado em 1924).

Freud, S. (2006). Josef Breuer. In Obras completas (v. XIX, pp. 299-300). Buenos Aires: Amorrortu. (Trabalho original publicado em 1925a).

Freud, S. (2004). Presentación autobiográfica. In Obras completas (v. XX, pp. 1-70). Buenos Aires: Amorrortu (Trabalho original publicado em 1925b).

Freud, S. (2006). Carta a Romain Rolland (Una perturbación del recuerdo en la Acrópolis). In Obras completas (v. XXII, pp. 209-221). Buenos Aires: Amorrortu. (Trabalho original publicado em 1936). 
Freud, S. (2005). Personajes psicopáticos en el escenario. In Obras completas (v. VII, pp. 273-282). Buenos Aires: Amorrortu. (Trabalho original publicado em 1942).

Freud, S. (1994). Cartas a Wilhelm Fliess (1887-1904). Buenos Aires: Amorrortu.

Freud, S. \& Jung, C. (1978). Correspondencia. Madrid: Taurus.

Jaspers, K. (2006). Psicopatología general. México: FCE. (Trabalho original publicado em 1913).

Lacan, J. (1981). El Seminario. Libro 1. Los escritos técnicos de Freud. Buenos Aires: Paidós. (Trabalho original publicado em 1953-1954).

Laplanche, J. \& Pontalis, J.-B. (1993). Diccionario de psicoanálisis. Barcelona: Paidós. Monedero, C. (1996). Psicopatología humana. Madrid: Siglo XXI.

Munguía, I.; Munguía, M. \& Rocha, G. (2000). Gramática. Lengua española. Reglas y ejercicios. México: Larousse.

Nicolescu, B. (1998). La transdisciplinariedad. Manifiesto. Monaco: Du Rocher.

Reuchlin, M. (1991). Historia de la psicología. México: Paidós.

Roudinesco, É. \& Plon, M. (2008). Diccionario de Psicoanálisis. $2^{\mathrm{a}}$ ed. Buenos Aires: Paidós.

Samacher, R. (2005). Psychologie clinique et psychopathologie. Paris: Bréal.

Singer, F. (2000, dez.). Psicopatología fundamental: de una cierta transmisión. Revista Latinoamericana de Psicopatologia Fundamental. São Paulo, 3(4), 112-121.

Tizón, J. (1978). Introducción a la epistemología de la psicopatología y la psiquiatría. Barcelona: Seix Barral.

Wallerstein, I. (1996). Abrir las ciencias sociales. Informe de la Comisión Gulbenkian para la reestructuración de las ciencias sociales. México: Siglo XXI/UNAM.

Zemelman, H. (1998). Acerca del problema de los límites disciplinarios. In I. Jaidar. Encrucijadas metodológicas en ciencias sociales (pp. 93-100). México: UAM-X.

\section{Resumos}

(Psicopatologia psicanalítica: um saber na encruzilhada)

A fim de esclarecer a situação epistêmica da psicopatologia psicanalítica no âmbito do saber freudiano, analisam-se questionamentos anteriores sobre o campo psicopatológico e suas possíveis modalidades relacionais com a psicanálise, a psicologia clínica e a psiquiatria. Os resultados destacam a originalidade da estratégia de Freud que, sob o nome de psicanálise, abriga um discurso racional sobre a experiência do sofrimento subjetivo.

Palavras-chave: Saber freudiano, psicopatologia, psicanálise, epistemologia 
(Psychoanalytic psychopathology: knowledge at the crossroads)

In order to clarify the epistemic status of psychoanalytic psychopathology in the framework of Freudian theory, previous questions in the psychopathological field are analyzed, together with their possible relational modalities and with psychoanalysis, clinical psychology and psychiatry. The results highlight the originality of Freud's strategy that, under the name of psychoanalysis, covers a rational discourse on the experience of subjects' discontent.

Key words: Freudian theory, psychopathology, psychoanalysis, epistemology

(Psychopathologie psychanalytique: un savoir au carrefour)

Afin de clarifier le statut épistémique de la psychopathologie psychanalytique dans le cadre du savoir freudien, cet article analyse des questions précédentes sur le domaine de la psychopathologie et ses possibles schémas relationnels avec la psychanalyse, la psychologie clinique et la psychiatrie. Les résultats soulignent l'originalité de la stratégie de Freud qui couvre, sous le nom de la psychanalyse, un discours rationnel sur l'expérience du malaise subjectif.

Mots clés: Savoir freudien, psychopathologie, psychanalyse, épistémologie

(Psychoanalytische Psychopathologie: ein Wissen am Scheideweg)

Mit dem Ziel, den epistemische Stand der psychoanalytischen Psychopathologie im Rahmen des freudschen Denkens zu klären, wurden von uns vorausgehende Fragen zum psychopathologischen Bereich und seine möglichen Beziehungsformen zur klinischen und psychiatrischen Psychoanalyse untersucht. Die Ergebnisse verdeutlichen Freuds strategische Originalität, der unter dem Begriff „Psychoanalyse“ einen rationalen Diskurs über die Erfahrung des subjektiven Leidens birgt.

Schlüsselwörter: freudsches Denken, Psychopathologie, Psychoanalyse, Epistemologie

Citação/Citation: Rubio. M. A. S. (2013, março). Psicopatología psicoanalítica: un saber en la encrucijada. Revista Latinoamericana de Psicopatologia Fundamental, 16(1), 56-70.

Editor do artigo/Editor: Prof. Dr. Manoel Tosta Berlinck

Recebido/Received: 27.10.2011/ 10.27.2011 Aceito/Accepted: 19.1.2012 / 1.19.2012

Copyright: (C) 2009 Associação Universitária de Pesquisa em Psicopatologia Fundamental/ University Association for Research in Fundamental Psychopathology. Este é um artigo de livre acesso, que permite uso irrestrito, distribuição e reprodução em qualquer meio, desde que 
o autor e a fonte sejam citados / This is an open-access article, which permits unrestricted use, distribution, and reproduction in any medium, provided the original author and source are credited.

Financiamento/Funding: $\mathrm{O}$ autor declara não ter sido financiado ou apoiado / The authors has no support or funding to report.

Conflito de interesses/Conflict of interest: $O$ autor declara que não há conflito de interesses / The author declares that has no conflict of interest.

\section{Miguel Angel Sierra Rubio}

Licenciado en Psicología por la Universidad Latina de México y Maestro en Psicología Clínica por la Universidad Autónoma de Querétaro. Socio-fundador de la Clínica Psicoterapéutica Sigmund Freud (México). Docente de pregrado y posgrado en la Universidad Latina de México, Facultad de Psicología.

Av. San José de Torres \# 100

Fracc. San José de Torres

C.P. 38085, Celaya, Gto., México

e-mail: miguel_sierra_7@hotmail.com 